\title{
Altered Expression of Angiogenic Biomarkers in Pregnancy Associated with Gestational Diabetes
}

\author{
Ebtisam Al-Ofi \\ Aziza Alrafiah (D) \\ Salman Maidi \\ Safa Almaghrabi \\ Nora Hakami \\ 'Department of Physiology, Faculty of \\ Medicine, King AbdulAziz University, \\ Jeddah, Saudi Arabia; ${ }^{2}$ Department of \\ Medical Laboratory Technology, Faculty \\ of Applied Medical Sciences, King \\ AbdulAziz University, Jeddah, Saudi \\ Arabia
}

Background: Gestational diabetes mellitus (GDM) typically occurs during the third trimester of pregnancy. Maternal hyperglycemic may influence the expression of pro-and antiangiogenic factors. Altered levels of angiogenic biomarkers in GDM pregnant women are associated with abnormal placentation. This study aimed to investigate the rates of expression of five angiogenic biomarkers called vascular endothelial growth factor-A (VEGF-A), angiopoietin-2, endoglin, endothelin-1, and granulocyte colony-stimulating factor (G-CSF) in GDM.

Methods: The samples were obtained from normal $(n=9)$ and GDM $(n=10)$ pregnancies. Multiplex assay was used to assess the levels of angiogenic biomarkers including VEGF-A, endoglin, endothelin-1, angiopoietin-2, and G-CSF in serum samples. All data were statistically analyzed using an unpaired Student's $t$-test. Correlations between measured parameters were made using Pearson correlations.

Results: VEGF-A, endoglin, endothelin-1, and angiopoietin-2 levels in GDM were significantly higher $(\mathrm{P}$ value $=0.001,0.042,0.049,0.001$; respectively) compared to control. However, G-CSF level exhibited a non-significant increase $(\mathrm{P}=0.466)$ in GDM compared to healthy controls. There was a significant positive correlation between angiopoietin-2 with endoglin, endothelin-1, and VEGF-A. Moreover, there was a significant positive correlation between VEGF-A with endoglin and endothelin-1. Most interestingly, there was a significant positive correlation between G-CSF with endothelin-1.

Conclusion: The angiogenic biomarkers were highly altered in pregnant women with GDM. The study provides a novel advance in the field of gestational diabetes, in terms of increase of angiogenic factors that can modify the vascularization of the placenta, the development of fetal vascular system and the insulin resistance itself.

Keywords: gestational diabetes mellitus, vascular endothelial growth factor A, angiopoietins, endoglin, endothelin-1, granulocyte colony-stimulating factor, pre-eclampsia

\section{Introduction}

Gestational diabetes mellitus (GDM) is described as glucose intolerance that manifests clinically in the second half of pregnancy. Approximately one in ten pregnant women worldwide are affected by GDM. ${ }^{1}$ It is associated with the placental vascular tree's morphological changes: increased surface area and capillary branching pregnancy. $^{2}$ So far, maternal hyperglycemia has been regarded as a significant mechanism for GDM-associated placental vascular alterations. Moreover, maternal hyperglycemic, pro-inflammatory conditions may also influence the expression of pro-and anti-angiogenic cytokines and growth factors. ${ }^{3}$
Correspondence: Aziza Alrafiah Department of Medical Laboratory Technology, Faculty of Applied Medical Sciences, King AbdulAziz University, PO Box 80200, Jeddah, 21589, Saudi Arabia Tel +966 I2640I000 Ext. 23495 Fax+966 I2640I000 Ext. 21686 Email aalrafiah@kau.edu.sa 
Angiogenesis is a physiological process that involves forming new blood vessels from the existing one in several steps, including vascular sprouting, tubule morphogenesis, and stabilization and adaption of the vessel. ${ }^{4}$ Hence, every step of the angiogenesis process targets regulation by paracrine, autocrine, and environmental factors. ${ }^{5}$ Feto-placental angiogenesis and vascular development are tightly regulated by the interaction between pro-and anti-angiogenic factors. A branched vascular network is essential for the placental formation and depends on these factors, including the vascular endothelial growth factor (VEGF), angiopoietin-1 (Ang-1), angiopoietin-2 (Ang-2), soluble endoglin (sEng), endothelin-1 (ET-1), and granulocyte colony-stimulating factor (G-CSF). ${ }^{6}$ Abnormal vasculature in the placenta is considered the most common placental pathology found in many pregnancy complications. ${ }^{7}$ Expression of the previous angiogenic biomarkers assists in vascular development and promotes angiogenesis for proper fetal development, regeneration, and wound healing. ${ }^{8}$

VEGF promotes angiogenesis in embryonic development and is essential in adults' wound healing. ${ }^{9}$ Any disruption in VEGF expression may lead to lethal outcomes resulting from abnormal blood vessel formation during embryogenesis. ${ }^{10}$ Nevertheless, Ang-2 may have a pro- or anti-angiogenic effect, depending on the presence of VEGF. ${ }^{11}$ In the absence of VEGF, Ang-2 activates endothelial cell apoptosis, vessel regression, and inhibits angiogenesis. However, in the presence of VEGF, Ang-2 works as an antagonist of Ang-1, destabilizes the interaction between endothelial cells and the supporting cells, induces vessel instability and the formation of disorganized and immature new blood vessels. ${ }^{12}$ Yet, endoglin is an anti-angiogenic molecule that has a significant role in pregnancy complications. ${ }^{13}$ Increased placental gene expression of ENG may lead to vascular dysfunction causing chronic fetal hypoxia, which may activate VEGF to increase angiogenesis as a feedback response to restore the fetus's placental circulation. ${ }^{14}$ In contrast to, stimulation of endothelin-1 A receptor via ET-1 that is accountable for vasoconstriction, activation of its endothelin-1 B receptor leads to vasodilation by enhanced nitric oxide development to meet the plasma volume expansion necessary for good pregnancy outcomes. However, increased stimulation or malfunction of the ET system in pregnant women is reported as resulting in hypertension leading to preeclampsia. ${ }^{15}$ In type 2 diabetes mellitus patients (T2DM), plasma levels of ET-1 are increasingly contributing to diabetes pathophysiology, ${ }^{16}$ which is ET-1 causes insulin resistance via direct action on the skeletal muscle rather than reducing insulin delivery resulting from vasoconstriction. ${ }^{15}$ Moreover, G-CSF plays a crucial role in pregnancy success by reducing pregnancy loss via activating the ovarian function and embryo implantation and promoting endometrial thickening. ${ }^{17}$ It is demonstrated that G-CSF: G-CSFR (specific receptor) interaction increases the secretion of VEGF, a factor that stimulates placental blood vessel formation. ${ }^{17,18}$

Alteration in paracrine angiogenic biomarkers may affect the feto-placental endothelium and may lead to gestational diabetes mellitus, and the subsequent maternal and neonatal complications, which include a high risk of developing preeclampsia, recurrent miscarriage, retinopathy, and an increase in infant birth weight. These, in turn, may contribute to GDM related changes in placental angiogenesis. ${ }^{2,3,19}$ Therefore, we hypothesized that angiogenic biomarkers could be altered in women with GDM and perhaps lead to maternal and neonatal complications and morbidities. To test this hypothesis, we compared the serum levels of five fundamental angiogenic biomarkers called VEGF, angiopoietin-2, endoglin, endothelin-1, and G-CSF in GDM and healthy pregnant women.

\section{Materials and Methods Ethical Approval}

The study was approved by the ethical committee of King Abdulaziz University Hospital (Reference No 336-16). Informed written consent was obtained from all participants. This study was conducted in accordance with the Declaration of Helsinki.

\section{Materials}

The Angiogenesis/Growth Factor Panel 1 AntibodyImmobilized Magnetic Beads kits (Anti-Human Angiopoietin-2 Bead (CatNo. HANGPT2-MAG), AntiHuman Endoglin Bead (CatNo. HENDGLN-MAG), Anti-Human Endothelin-1 Bead (CatNo. HET1-MAG), Anti-Human G-CSF Bead (CatNo. HAGGCSF-MAG), and Anti-Human VEGF-A Bead (CatNo. HVEGFMAG) were purchased from Millipore Corp (USA). The standards (CatNo. HNDG2-8036-2), quality 
controls 1 and 2 (CatNo. HNDG2-6036-2), bead diluent (CatNo. LBD), detection antibodies (CatNo. HNDG21036-2), and streptavidin-phycoerythrin (CatNo. L-SAPE6) were purchased from Invitrogen.

\section{Sample Collection and Processing}

Venous whole blood was collected by venipuncture from nine healthy pregnant women (aged 20-40 years) with no previously or currently diagnosed GDM and ten pregnant women with GDM (aged 20-40 years). GDM was diagnosed on an oral glucose tolerance test of $75 \mathrm{~g}$ following the current IADPSG guidelines..$^{20}$ Subjects with bleeding disorders, coagulation disorders, or those who are on anticoagulant therapy were excluded.

Blood was collected into plane tubes contain no anticoagulants. The blood was allowed to clot for at least 30 minutes before centrifugation for 10 minutes at $1000 \times \mathrm{g}$ to obtain serum, which was aliquoted and stored at $-80^{\circ} \mathrm{C}$ to be used later in the determination of angiogenic biomarkers concentrations. ${ }^{21}$ The procedure of measuring angiogenic biomarkers is based on antigen-antibody reaction on immunoassay machines called Luminex 200 (Luminex 100/200, USA).

\section{Methods}

The angiogenic biomarkers concentrations in the serum were measured using multiplex xMAP technology on a Luminex 200 instrument with commercially available panels from Millipore Corporation (Billerica, MA, USA). The procedures were performed according to the manufacturer's instructions, and the control samples that were provided within the kits were assayed in each analysis, and samples were run in duplicate. In our study, the levels of VEGF-A, endoglin, endothelin-1, angiopoietin-2, and G-CSF were studied. The data were generated by the 3.1 Xponent software package (Luminex Corporation, USA).

\section{Statistical Analysis}

The data were analyzed using version 7 GraphPad Prism software. All data were statistically analyzed using an unpaired Student's $t$-test. Correlations between measured parameters were made using Pearson correlations. P values less than 0.05 were considered significant. Figures were generated using GraphPad Prism (version 7; San Diego, USA).

\section{Results}

\section{Clinical Characteristics of Pregnant} Women Participating in the Present Study

All participants in the present study were recruited from $\mathrm{KAUH}$, at their routine antenatal clinic before any diabetic therapy was administrated, the regardless diagnosis was confirmed later, and they were distributed into the GDM group or healthy pregnant group. Maternal age, gestational age (weeks), and other gestational-related data were collected from ten GDM women and compared to nine normal pregnant (NP) women. Clinical characteristics of the study participants are presented in Table 1. The study participants' clinical characteristics, including age, gestational age at GDM diagnosis, gravidity, parity, abortion, intrauterine fetal death, and body mass index, were non-significantly different between GDM groups and the healthy participants.

\section{Comparison of Angiopoietin-2 (Pg/Ml) Levels Between Control Pregnant and GDM-Samples}

Angiopoietin-2 serum levels in GDM women were significantly higher compared to controls healthy pregnant women (healthy vs GDM; $3769.67 \pm 374.19 \mathrm{pg} / \mathrm{mL}$ vs $15,343.90$ $\pm 807.90 \mathrm{pg} / \mathrm{mL}, \mathrm{P}=0.001$ ) as shown in (Figure 1).

Table I Clinical Characteristics of Pregnant Women Participating in the Present Study

\begin{tabular}{|l|c|c|c|}
\hline Variables & GDM (n= 10) & NP (n= 9) & P-value \\
\hline Age (years) & $32.7 \pm 7.1$ & $33 \pm 8$ & NS \\
\hline $\begin{array}{l}\text { Gestational age } \\
\text { (weeks) }\end{array}$ & $26.4 \pm 2.7$ & $25.5 \pm 1.8$ & NS \\
\hline Gravidity & $4.8 \pm 3.1$ & $4.6 \pm 3.6$ & NS \\
\hline Parity & $2.1 \pm 1.7$ & $3.7 \pm 2$ & NS \\
\hline Abortion or IUFD & $2.7 \pm 1.4$ & $0.9 \pm 1.6$ & NS \\
\hline BMI & $30.5 \pm 5$ & $28 \pm 5.7$ & NS \\
\hline RBS & $7.8 \pm 2.5$ & $4.6 \pm 0.7$ & 0.013 \\
\hline $\begin{array}{l}\text { GTT } \\
\text { Fasting } \\
2 \mathrm{hrs}\end{array}$ & $\begin{array}{c}5.6 \pm 0.8 \\
9.8 \pm 2.5\end{array}$ & - & \\
\hline
\end{tabular}

Abbreviations: BMI, body mass index; GDM, gestational diabetes mellitus; IUFD, intrauterine fetal death; NS, not significant; RBS, random blood sugar; GTT, glucose tolerance test. 


\section{Comparison of VEGF-A (Pg/MI) Levels \\ Between Control Pregnant and GDM-Samples}

The serum level of VEGF-A in the GDM group was significantly higher $(\mathrm{P}=0.001)$ compared to the healthy controls (healthy vs GDM; $146.60 \pm 12.03 \mathrm{pg} / \mathrm{mL}$ vs 296.92 $\pm 17.37 \mathrm{pg} / \mathrm{mL}, \mathrm{P}=0.001$ ) (Figure 2).

\section{Comparison of Endoglin (Pg/MI) Levels Between Control Pregnant and GDM-Samples}

Furthermore, the difference in endoglin concentration in serum between GDM women and healthy participants was significant (healthy vs GDM; $1444.78 \pm 82.30 \mathrm{pg} / \mathrm{mL}$ vs $1814.06 \pm 141.10 \mathrm{pg} / \mathrm{mL}, \mathrm{P}=0.042$ ) (Figure 3).

\section{Comparison of Endothelin (Pg/MI) Levels Between Control Pregnant and GDM-Samples}

Endothelin-1 concentration in the GDM participants $(6.58$ $\pm 0.13 \mathrm{pg} / \mathrm{mL})$ was significantly higher $(\mathrm{P}=0.049)$ in comparison to the healthy group endothelin-1 level $(6.12 \pm 0.17 \mathrm{pg} /$ $\mathrm{mL}$ ) (Figure 4). However, G-CSF serum level non significantly ( $\mathrm{P}=0.466$ ) increased in the GDM group (healthy vs GDM; $124.61 \pm 2.12 \mathrm{pg} / \mathrm{mL}$ vs $129.46 \pm 5.86 \mathrm{pg} / \mathrm{mL}$ ), but to

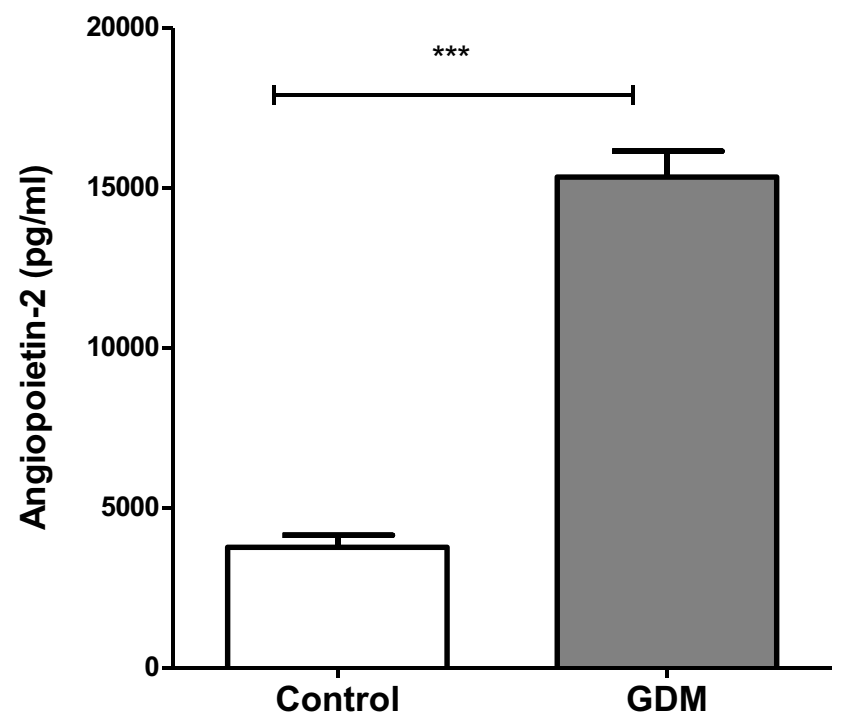

Figure I Comparison of angiopoietin-2 $(\mathrm{pg} / \mathrm{mL})$ levels between control pregnant and GDM-samples. Data are expressed as mean \pm standard error. $* * * \mathrm{P}=0.001$ compared to control.

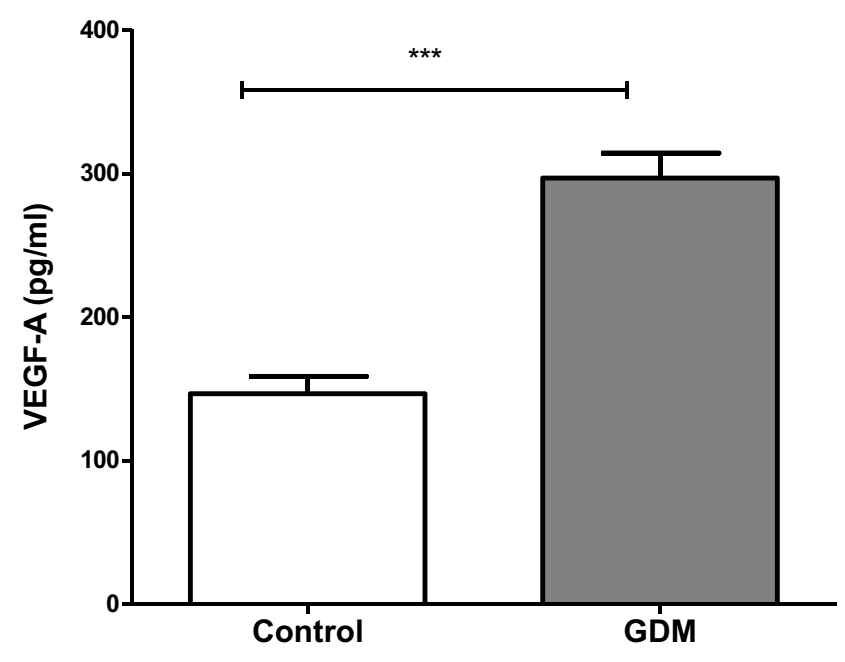

Figure 2 Comparison of VEGF-A ( $\mathrm{pg} / \mathrm{mL})$ levels between control pregnant and GDM-samples. Data are expressed as mean \pm standard error. $* * * \mathrm{P}=0.00 \mathrm{I}$ compared to control.

a lesser extent compares to the rest of the angiogenic biomarkers.

\section{Correlations Between Angiopoietin-2 and Endoglin in Control}

In the control group, only angiopoietin-2 exhibited a significant positive correlation with endoglin, using the Pearson productmoment correlation test (Figure 5). However, the other studied biomarkers did not demonstrate significant correlations within the control group (data unshown).

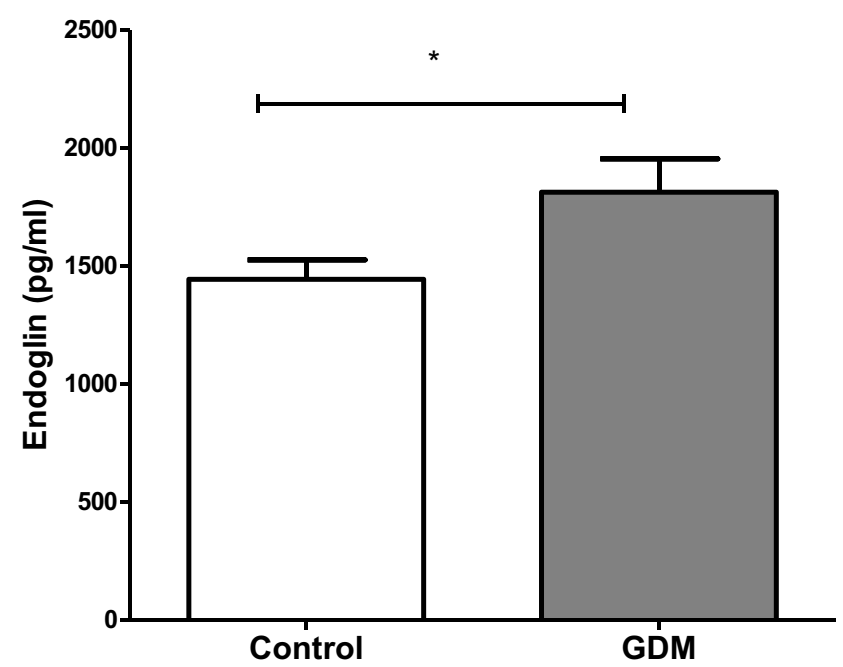

Figure 3 Comparison of endoglin $(\mathrm{pg} / \mathrm{mL})$ levels between control pregnant and GDM-samples. Data are expressed as mean \pm standard error. $* \mathrm{P}<0.05$ compared to control. 


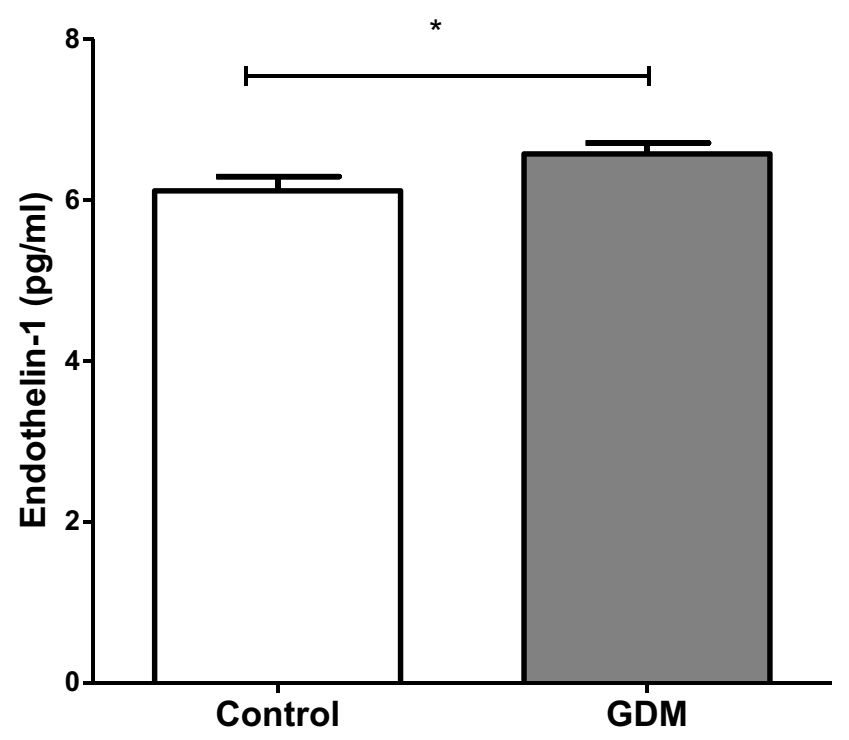

Figure 4 Comparison of endothelin $(\mathrm{pg} / \mathrm{mL})$ levels between control pregnant and GDM-samples. Data are expressed as mean \pm standard error. $* \mathrm{P}<0.05$ compared to control.

\section{Correlations Between Angiopoietin-2 with Endoglin, Endothelin-I, and VEGF- $A$ in GDM Patients}

In the GDM patients: endoglin, endothelin-1 and VEGFA showed significant positive correlations $(\mathrm{r}=0.868$, $\mathrm{P}=0.001), \quad(\mathrm{r}=0.729, \quad \mathrm{P}=0.017) \quad$ and $\quad(\mathrm{r}=0.964$, $\mathrm{P}=0.001$ ), respectively with serum level parameter of angiopoietin-2 (Figure 6).

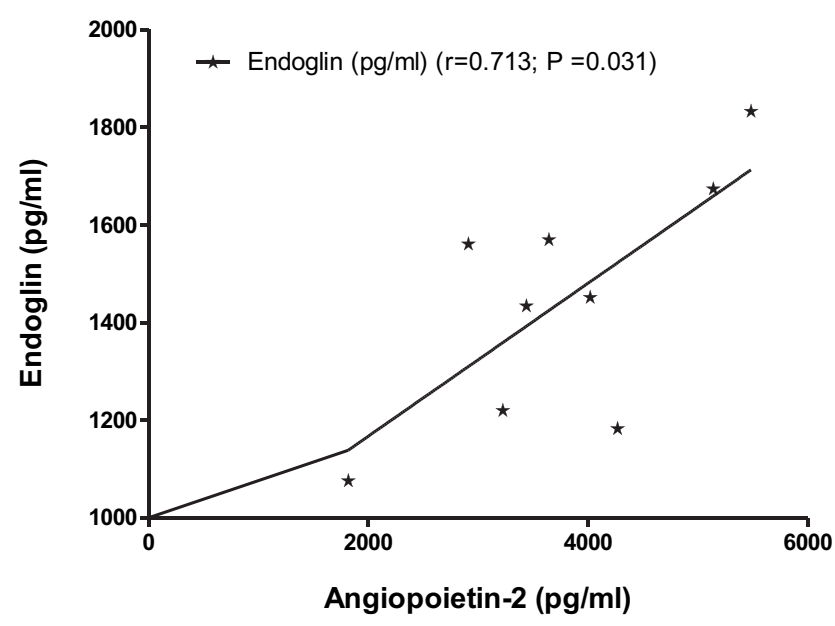

Figure 5 Correlations between endoglin and angiopoietin -2 in control group. The correlation coefficient was made using the Person test.

\section{Correlations Between G-CSF and Endothelin-I in GDM Patients}

Despite of a reciprocal small sample size, the G-CSF exhibited significant positive correlations with the endothelin-1 parameter $(\mathrm{r}=0.667, \mathrm{P}=0.035)$ (Figure 7). Interestingly, endoglin and endothelin-1 exhibited positive significant correlations $(\mathrm{r}=0.729, \mathrm{P}=0.017)$ and $(\mathrm{r}=0.706$, $\mathrm{P}=0.023$ ); respectively with the VEGF-A (Figure 8).

\section{Discussion}

The current study demonstrated that the serum level of angiogenic biomarkers (VEGF, angiopoietin-2, endoglin, and endothelin-1) in pregnant women with GDM were significantly higher compared to healthy pregnant women. However, G-CSF level was high in GDM patients, but the result was not significant compared to normal participants.

Pathological changes in maternal blood properties (such as hypoxia or hyperglycemia); or pro-and anti-angiogenic factors such as VEGFs and soluble endoglin may directly affect the growth and function of feto-placental vessels. Alterations in the feto-placental vessels may be associated with the pathogenesis of GDM.

Expression of the angiogenic biomarkers helps in vascular development and promotes angiogenesis for proper fetal development. ${ }^{8}$ Any alteration in angiogenic biomarkers' levels is associated with abnormal placentation and the subsequent maternal and neonatal complications. ${ }^{19}$

In the present study, the GDM group showed a significant increase in serum VEGF-A levels than healthy pregnant women. The results presented here are similar to those of another recent study that investigated GDM rats and showed a significant increase in serum VEGF-A level and advanced glycation end-products (AGEs) concentrations. ${ }^{22}$ The potential mechanism(s) for increase VEGF-A level might be that AGEs perform their harmful effects, whether by actively destroying cells or by binding to the receptor of advanced glycation end-products (RAGE). As binding AGEs with RAGE enhances activation of the NF-kB pathway and increases VEGF production, rendering this significant mechanism underlying malfunction at the placental barrier of GDM. ${ }^{23}$ Another potential mechanism is that elevated maternal hemoglobin A1c (HbA1c) levels may result in a discrepancy in supply and demand for oxygen, resulting in a temporary decrease in fetal oxygen levels (ie, hypoxia). Transient fetal hypoxia can cause increased placental synthesis and VEGF release, as well as VEGF receptor expression. ${ }^{1}$ 

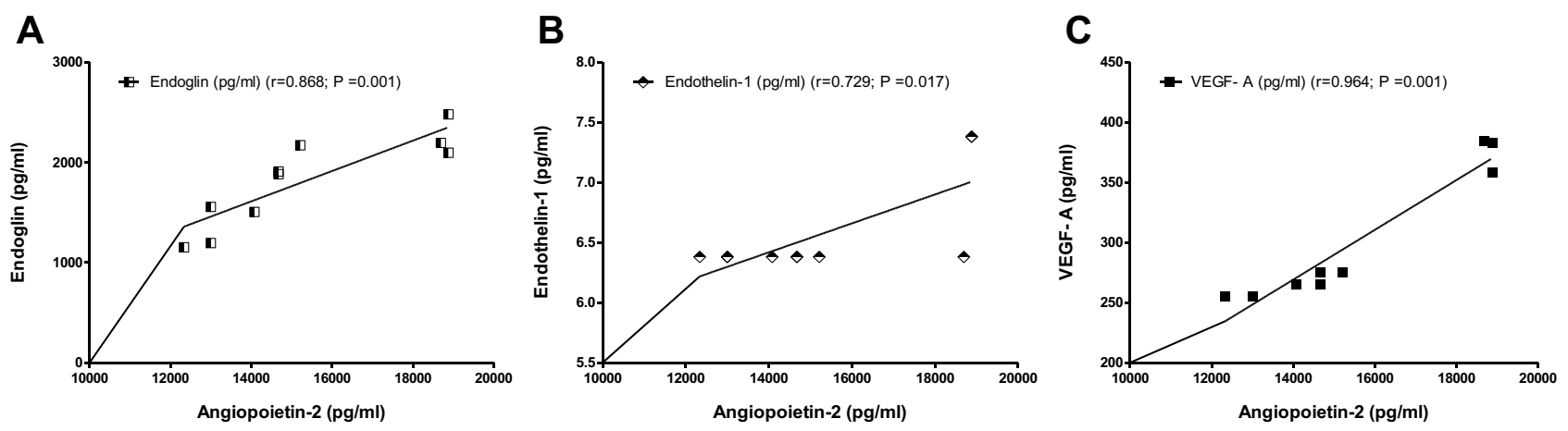

Figure 6 Correlations between angiopoietin -2 and endoglin (A), angiopoietin -2 and endothelin-I (B) and angiopoietin -2 and VEGF- A (C) in GDM patients. Correlation coefficient was made using Pearson test.

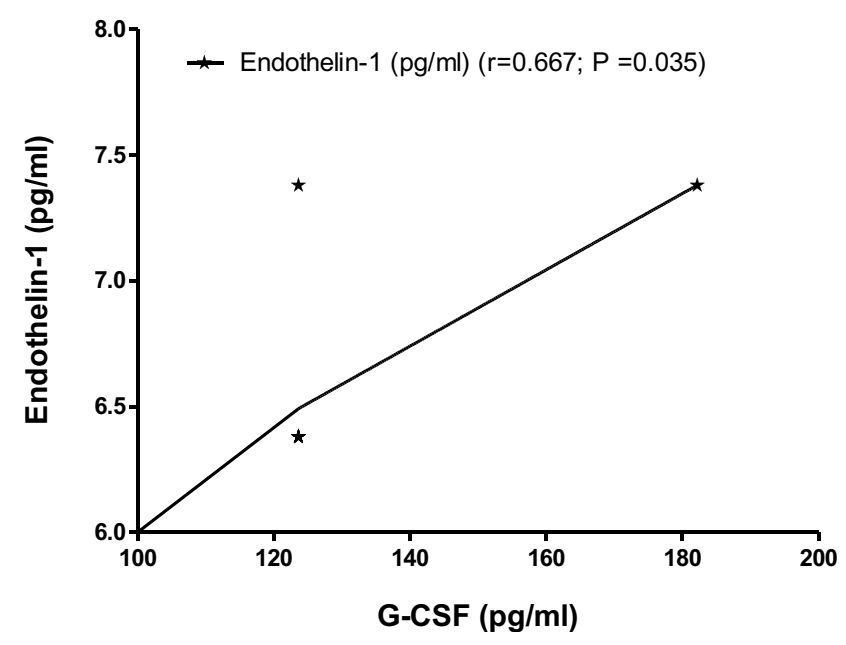

Figure 7 Correlations between G-CSF and endothelin-I in GDM patients. The correlation coefficient was made using the Pearson test.

However, our results contrast to another study that demonstrated decreased concentrations of VEGF-A and

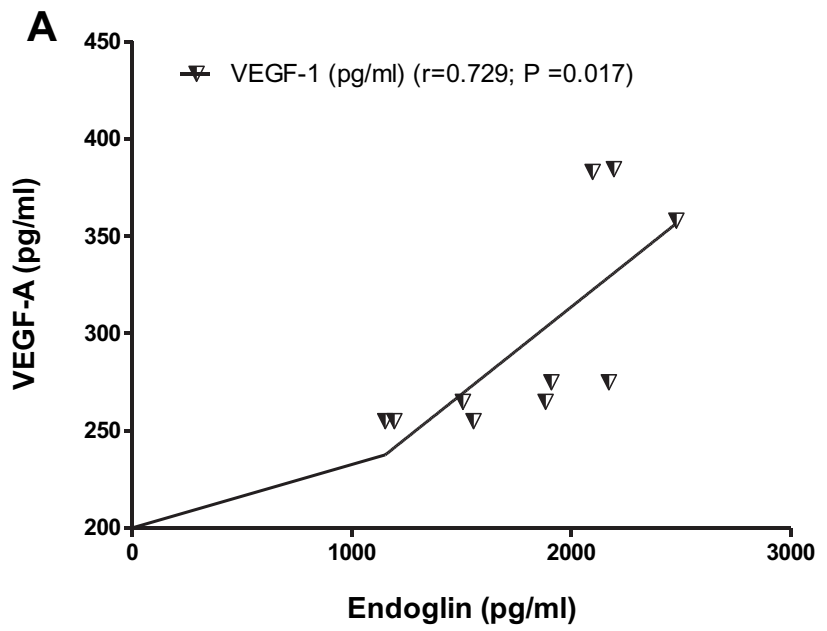

VEGF receptor in placental tissues collected from GDM females compared to healthy pregnant women. ${ }^{24}$ The explanation for these conflicting reports may be due to differences in the sample as Meng et al (2016) used placental tissue samples while in our study, we measured the serum level of VEGF-A. The discrepancy between the VEGF-A concentration between placental tissues and serum could be a pathogenic disease feature.

Furthermore, we have found that Angiopoietin-2 serum concentrations significantly increased in GDM patients compared to the healthy group. Similar results were observed in previous research and reported a significant positive correlation between angiopoietin with insulin resistance in the GDM group and concluded that serum angiopoietin level could be a predictor of GDM. ${ }^{25}$

In particular, the VEGF-A mechanism of action tends to be similar to the angiopoietin-2, especially in the late stage of angiogenesis. They act through distinct tyrosine kinase receptors, which indicates that VEGF-A has

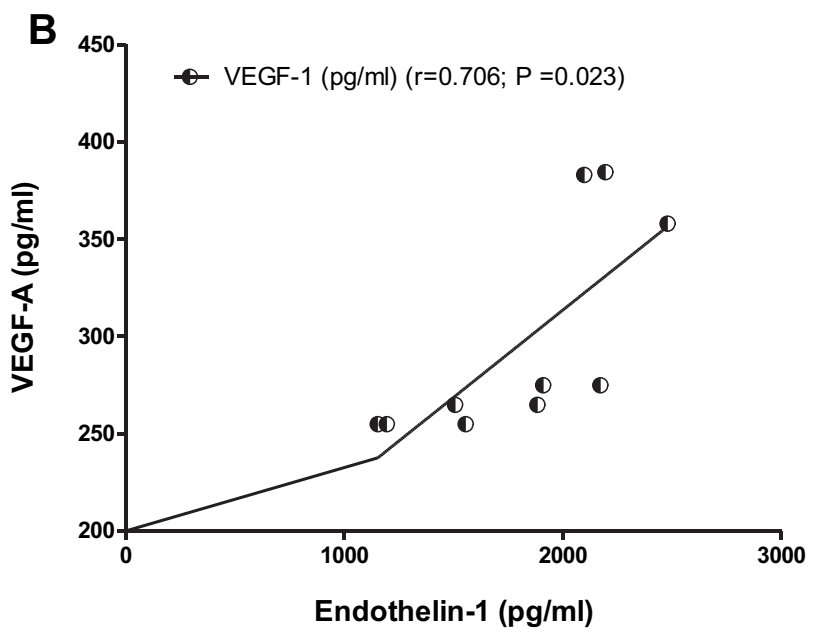

Figure 8 Correlations between endoglin and VEGF-A (A) and endothelin-I and VEGF-A (B) in GDM patients. The correlation coefficient was made using the Pearson test. 
indirectly affected the angiopoietins functions by the proteolytic shedding of the angiopoietin receptor. ${ }^{26,27}$ That could explain our findings that there was a significant positive correlation between the angiopoietin serum level and VEGF in the GDM group.

In the current study, the GDM group showed a significant increase in endoglin serum concentrations than the healthy pregnant group. A similar finding was shown in pregnant women with T1DM relative to those of non-diabetic women, which may lead to the raised prevalence of preeclampsia and interfere with the development of blood vessel complications. ${ }^{28}$ Additionally, we have found that serum level of VEGF-A significant correlations endoglin, which is in agreement with Masuyama et al (2007) study. ${ }^{29}$

Moreover, the endothelin-1 serum concentrations were significantly higher in the GDM pregnant participants. Similar results were reported in patients with T1DM and T2DM, and it has been suggested that changes in plasma endothelin-1 levels may precede vascular complications associated with hypertension and diabetes. ${ }^{30}$ It has been found the human placenta expresses endothelin-1, and its circulating levels are high in pregnancies suffering from GDM. ${ }^{31}$ In contrast, two previous studies reported a normal endothelin-1 plasma level in women with GDM. ${ }^{32,33}$

In the present study, the GDM group showed a nonsignificant increase in G-CSF serum concentrations than the healthy control group. Previous research showed similar results that the serum G-CSF rates among diabetes subjects were significantly higher than those without diabetes; also the diabetic group that used insulin and oral hypoglycemic agents, their serum G-CSF levels were lower. ${ }^{34}$ However, our results contrast to another study that G-CSF level decreased in patients with T1DM or T2DM compared to control. ${ }^{35}$ G-CSF can play a significant role in stimulating vascular endothelium injury and granulocytes, which may play a significant role in the development of preeclampsia. ${ }^{36}$ That effect could explain the significant positive correlation in the current study between the G-CSF and the endothelin-1 as the endothelin-1, known as a potent vasoconstrictor. It could interfere with the normal angiogenesis function of diabetic mothers by blocking off some angiogenic biomarkers such as G-CSF.

\section{Conclusion}

The present study showed that in GDM pregnant women, the level of these factors VEGF, angiopoietin-2, endoglin, and endothelin-1 are significantly higher than in control. Further research is warranted to understanding the association and interaction between altered levels of angiogenic factors and pregnancy associated with gestational diabetes and investigated their suitability for therapeutic and diagnostic applications.

\section{Institutional Review Board Statement}

The study was conducted according to the guidelines of the bioethics and research committee of the King Abdulaziz University (KAUH), Jeddah, Saudi Arabia, and approved by the Institutional Scientific and Research Committee under the reference number of (Reference No 336-16).

\section{Acknowledgment}

This manuscript is a Master's degree thesis that has been submitted to the Deanship of Graduate Studies, King Abdulaziz University.

\section{Funding}

This research received no external funding.

\section{Disclosure}

The authors declare no conflicts of interest for this work.

\section{References}

1. Troncoso F, Acurio J, Herlitz K, et al. Gestational diabetes mellitus is associated with increased pro-migratory activation of vascular endothelial growth factor receptor 2 and reduced expression of vascular endothelial growth factor receptor 1. PLoS One. 2017;12(8): e0182509. doi:10.1371/journal.pone.0182509

2. Loegl J, Nussbaumer E, Cvitic S, Huppertz B, Desoye G, Hiden U. GDM alters paracrine regulation of feto-placental angiogenesis via the trophoblast. Lab Invest. 2017;97(4):409-418. doi:10.1038/ labinvest.2016.149

3. Sobrevia L, Salsoso R, Fuenzalida B, et al. Insulin Is a Key Modulator of Fetoplacental Endothelium Metabolic Disturbances in Gestational Diabetes Mellitus. Front Physiol. 2016;7:119. doi:10.3389/ fphys.2016.00119

4. Betz C, Lenard A, Belting HG, Affolter M. Cell behaviors and dynamics during angiogenesis. Development. 2016;143 (13):2249-2260. doi:10.1242/dev.135616

5. Conway EM, Collen D, Carmeliet P. Molecular mechanisms of blood vessel growth. Cardiovasc Res. 2001;49(3):507-521. doi:10.1016/ S0008-6363(00)00281-9

6. Umapathy A, Chamley LW, James JL. Reconciling the distinct roles of angiogenic/anti-angiogenic factors in the placenta and maternal circulation of normal and pathological pregnancies. Angiogenesis. 2020;23 (2):105-117. doi:10.1007/s10456-019-09694-w

7. Chen DB, Zheng J. Regulation of placental angiogenesis. Microcirculation. 2014;21(1):15-25. doi:10.1111/micc.12093 
8. Suganya N, Bhakkiyalakshmi E, Sarada DV, Ramkumar KM. Reversibility of endothelial dysfunction in diabetes: role of polyphenols. $\quad B r \quad J \quad$ Nutr. 2016;116(2):223-246. doi:10.1017/ S0007114516001884

9. Carmeliet P. VEGF as a key mediator of angiogenesis in cancer. Oncology. 2005;69(Suppl 3):4-10. doi:10.1159/000088478

10. Padmanabhan S, Lee VW, McLean M, et al. The Association of Falling Insulin Requirements With Maternal Biomarkers and Placental Dysfunction: a Prospective Study of Women With Preexisting Diabetes in Pregnancy. Diabetes Care. 2017;40 (10):1323-1330. doi:10.2337/dc17-0391

11. Li L, Juan-Yu H, Jian-Yong $X$, et al. Association between interleukin-19 and angiopoietin-2 with vascular complications in type 2 diabetes. J Diabetes Investig. 2016;7(6):895-900.

12. Melincovici CS, Bosca AB, Susman S, et al. Vascular endothelial growth factor (VEGF) - key factor in normal and pathological angiogenesis. Rom J Morphol Embryol. 2018;59(2):455-467.

13. Bus P, Gerrits T, Heemskerk SAC, et al. Endoglin Mediates Vascular Endothelial Growth Factor-A-Induced Endothelial Cell Activation by Regulating Akt Signaling. Am J Pathol. 2018;188(12):2924-2935. doi:10.1016/j.ajpath.2018.08.005

14. Szentpeteri I, Rab A, Kornya L, Kovacs P, Brubel R, Joo JG. Placental gene expression patterns of endoglin (CD105) in intrauterine growth restriction. J Matern Fetal Neonatal Med. 2014;27 (4):350-354. doi:10.3109/14767058.2013.818125

15. Gillis EE, Sasser JM, Sullivan JC. Endothelin, sex, and pregnancy: unique considerations for blood pressure control in females. Am $J$ Physiol Regul Integr Comp Physiol. 2016;310(8):R691-6. doi:10.1152/ajpregu.00427.2015

16. Horinouchi T, Hoshi A, Harada T, et al. Endothelin-1 suppresses insulin-stimulated Akt phosphorylation and glucose uptake via GPCR kinase 2 in skeletal muscle cells. Br J Pharmacol. 2016;173 (6):1018-1032. doi:10.1111/bph.13406

17. Eftekhar M, Naghshineh E, Khani P. Role of granulocyte colony-stimulating factor in human reproduction. $J$ Res Med Sci. 2018;23(1):7. doi:10.4103/jrms.JRMS_628_17

18. Lacko LA, Hurtado R, Hinds S, Poulos MG, Butler JM, Stuhlmann H. Altered feto-placental vascularization, feto-placental malperfusion and fetal growth restriction in mice with Egfl7 loss of function. Development. 2017;144(13):2469-2479. doi:10.1242/ dev. 147025

19. Rizov M, Andreeva P, Dimova I. Molecular regulation and role of angiogenesis in reproduction. Taiwan J Obstet Gynecol. 2017;56 (2):127-132. doi:10.1016/j.tjog.2016.06.019

20. Chi C, Loy SL, Chan SY, et al. Impact of adopting the 2013 World Health Organization criteria for diagnosis of gestational diabetes in a multi-ethnic Asian cohort: a prospective study. BMC Pregnancy Childbirth. 2018;18(1):69. doi:10.1186/s12884-018-1707-3

21. Geurtsen ML, van Soest EEL, Voerman E, Steegers EAP, Jaddoe VWV, Gaillard R. High maternal early-pregnancy blood glucose levels are associated with altered fetal growth and increased risk of adverse birth outcomes. Diabetologia. 2019;62(10):1880-1890. doi:10.1007/s00125-019-4957-3

22. Shi Y, Qian J, Zhang F, et al. Low molecular weight heparin (nadroparin) improves placental permeability in rats with gestational diabetes mellitus via reduction of tight junction factors. Mol Med Rep. 2020;21(2):623-630. doi:10.3892/mmr.2019.10868
23. Boulanger E, Grossin N, Wautier MP, Taamma R, Wautier JL. Mesothelial RAGE activation by AGEs enhances VEGF release and potentiates capillary tube formation. Kidney Int. 2007;71(2):126-133. doi:10.1038/sj.ki.5002016

24. Meng Q, Shao L, Luo X, et al. Expressions of VEGF-A and VEGFR-2 in placentae from GDM pregnancies. Reprod Biol Endocrinol. 2016;14(1):61. doi:10.1186/s12958-016-0191-8

25. Abdullah B, Deveci K, Atilgan R, Kilicli F, Soylemez MS. Serum angiopoietin-related growth factor (AGF) levels are elevated in gestational diabetes mellitus and associated with insulin resistance. Ginekol Pol. 2012;83(10):749-753.

26. Findley CM, Cudmore MJ, Ahmed A, Kontos CD. VEGF induces Tie2 shedding via a phosphoinositide 3-kinase/Akt dependent pathway to modulate Tie2 signaling. Arterioscler Thromb Vasc Biol. 2007;27(12):2619-2626. doi:10.1161/ATVBAHA.107.150482

27. Kappou D, Sifakis S, Konstantinidou A, Papantoniou N, Spandidos DA. Role of the angiopoietin/Tie system in pregnancy (Review). Exp Ther Med. 2015;9(4):1091-1096. doi:10.3892/ etm.2015.2280

28. Yu Y, Jenkins AJ, Nankervis AJ, et al. Anti-angiogenic factors and pre-eclampsia in type 1 diabetic women. Diabetologia. 2009;52 (1):160-168. doi:10.1007/s00125-008-1182-x

29. Masuyama H, Nakatsukasa H, Takamoto N, Hiramatsu Y. Correlation between soluble endoglin, vascular endothelial growth factor receptor-1, and adipocytokines in preeclampsia. J Clin Endocrinol Metab. 2007;92(7):2672-2679. doi:10.1210/jc.2006-2349

30. Schneider JG, Tilly N, Hierl T, et al. Elevated plasma endothelin-1 levels in diabetes mellitus. Am J Hypertens. 2002;15(11):967-972. doi:10.1016/S0895-7061(02)03060-1

31. Dieber-Rotheneder M, Beganovic S, Desoye G, Lang U, CervarZivkovic M. Complex expression changes of the placental endothelin system in early and late onset preeclampsia, fetal growth restriction and gestational diabetes. Life Sci. 2012;91(13-14):710-715. doi:10.1016/j.lfs.2012.04.040

32. Swiderski S, Celewicz Z, Miazgowski T, Ogonowski J. Maternal endothelin-1 and cyclic guanosine monophosphate concentrations in pregnancies complicated by pregravid and gestational diabetes mellitus. Gynecol Obstet Invest. 2010;69(1):46-50. doi:10.1159/ 000253851

33. Telejko B, Zonenberg A, Kuzmicki M, et al. Circulating asymmetric dimethylarginine, endothelin-1 and cell adhesion molecules in women with gestational diabetes. Acta Diabetol. 2009;46 (4):303-308. doi:10.1007/s00592-008-0088-x

34. Surendar J, Mohan V, Pavankumar N, Babu S, Aravindhan V. Increased levels of serum granulocyte-macrophage colony-stimulating factor is associated with activated peripheral dendritic cells in type 2 diabetes subjects (CURES-99). Diabetes Technol Ther. 2012;14(4):344-349. doi:10.1089/dia.2011.0182

35. Alaa HAO, Hussein D, Raheem M, Hasanain S, Noor K. Impact of Serum Granulocyte-Macrophage Colony-Stimulating Factor Levels among Diabetes Patients in Hilla City - Iraq. Adv Life Sci. 2014;4 (6):260-264. doi:10.5923/j.als.20140406.02

36. Giaglis S, Stoikou M, Sur Chowdhury C, et al. Multimodal Regulation of NET Formation in Pregnancy: progesterone Antagonizes the Pro-NETotic Effect of Estrogen and G-CSF. Front Immunol. 2016;7:565. doi:10.3389/fimmu.2016.00565 


\section{Publish your work in this journal}

The International Journal of General Medicine is an international, peer-reviewed open-access journal that focuses on general and internal medicine, pathogenesis, epidemiology, diagnosis, monitoring and treatment protocols. The journal is characterized by the rapid reporting of reviews, original research and clinical studies across all disease areas. The manuscript management system is completely online and includes a very quick and fair peer-review system, which is all easy to use. Visit http://www.dovepress.com/ testimonials.php to read real quotes from published authors.

Submit your manuscript here: https://www.dovepress.com/international-journal-of-general-medicine-journal 